\title{
Blockage of Drp1 phosphorylation at Ser579 protects neurons against $\mathbf{A} \boldsymbol{\beta}_{1-42}$-induced degeneration
}

\author{
DAN XU ${ }^{1,2^{*}}$, PING YANG $^{3 *}$, ZHANG-JIAN YANG ${ }^{4}$, QIU-GEN LI $^{1}$, YE-TONG OUYANG ${ }^{3}$, TING YU $^{3}$, \\ JIAN-HUI SHANGGUAN ${ }^{3}$, YU-YING WAN ${ }^{5}$, LI-PING JIANG ${ }^{4}$, XIN-HUI QU ${ }^{1,3}$ and XIAO-JIAN HAN ${ }^{1,3,6}$ \\ ${ }^{1}$ Institute of Geriatrics, Affiliated People's Hospital of Nanchang University, Nanchang, Jiangxi 330006; \\ ${ }^{2}$ Department of Ophthalmology, Southern Medical University Pingxiang Hospital, Pingxiang, Jiangxi 337000; \\ ${ }^{3}$ Department of Neurology, Affiliated People's Hospital of Nanchang University; ${ }^{4}$ Department of Pharmacology, \\ School of Pharmaceutical Science, Nanchang University; ${ }^{5}$ Department of Intra-Hospital Infection Management, \\ The Second Affiliated Hospital of Nanchang University; ${ }^{6}$ Research Institute of Ophthalmology and Visual Sciences, \\ Affiliated Eye Hospital of Nanchang University Nanchang, Jiangxi 330006, P.R. China
}

Received March 14, 2021; Accepted June 24, 2021

DOI: $10.3892 / \mathrm{mmr} .2021 .12296$

\begin{abstract}
Alzheimer's disease (AD), one of the most common types of chronic neurodegenerative diseases, is pathologically characterized by the formation of amyloid $\beta$ $(\mathrm{A} \beta)$ peptide-containing plaques and neurofibrillary tangles. Among $A \beta$ peptides, $A \beta_{1-42}$ induces neuronal toxicity and neurodegeneration. In our previous studies, Cdk5 was found to regulate $\mathrm{A} \beta_{1-42}$-induced mitochondrial fission via the phosphorylation of dynamin-related protein 1 (Drp1) at Ser579. However, whether blockage of Drp1 phosphorylation at Ser579 protects neurons against $\mathrm{A} \beta_{1-42}$-induced degeneration remains to be elucidated. Thus, the aim the present study was to examine the effect of mutant Drp1-S579A on neurodegeneration and its underlying mechanism. First, the phosphorylation-defect (phospho-defect) mutant, Lenti-Drp1-S579A was constructed. Phospho-defect Drp1-S579A expression was detected in primary cultures of mouse cortical neurons infected with Lenti-Drp1-S579A using western blotting and it was found to successfully attenuate the phosphorylation of endogenous Drp1 at Ser579. In primary neuronal cultures, the neuronal processes were evaluated under microscopy. Treatment with $10 \mu \mathrm{M}$ $\mathrm{A} \beta_{1-42}$ significantly decreased dendritic density and length, spine outgrowth and synapse number. As expected, infection of neurons with Lenti-Drp1-S579A efficiently alleviated the
\end{abstract}

Correspondence to: Professors Xiao-Jian Han or Xin-Hui Qu, Institute of Geriatrics, Affiliated People's Hospital of Nanchang University, 152 Aiguo Road, Nanchang, Jiangxi 330006, P.R. China E-mail: hanxiaojian@hotmail.com

E-mail: quxh012543@163.com

${ }^{*}$ Contributed equally

Key words: amyloid $\beta_{-42}$, dynamin-related protein $1, \mathrm{Cdk} 5$, neurodegeneration, apoptosis inhibitory effect of $A \beta_{1-42}$ on neurite outgrowth and synapse density. In addition, infection with Lenti-Drp1-S579A abolished the cleavage of caspase- 3 and apoptosis in neurons exposed to $\mathrm{A} \beta_{1-42}$. Thus, the current data demonstrated that blockage of Drp1 phosphorylation at Ser579 may be an effective strategy to protect neurons against $\mathrm{A} \beta_{1-42}$-induced degeneration and apoptosis. These findings underline the therapeutic potential of targeting Drp1 in the treatment of AD.

\section{Introduction}

Alzheimer's disease (AD) is an age-related, progressive neurodegenerative disease, with clinical symptoms of cognitive decline and changes in behavior and personality $(1,2)$. AD is the commonest type of dementia in people aged $>65$ (3). With the increase in human lifespan $\mathrm{AD}$ is becoming, at present, a major health concern for the elderly. Worldwide $>50$ million people are suffering from AD (3). However, there is no effective treatment for delaying the disease progression of patients with AD (4). The disease is characterized by two hallmark lesions: Senile plaques (SP) and neurofibrillary tangles (NFTs). SP and NFTs mainly result from the deposition of amyloid $\beta$ (A $\beta$ ) peptides and hyperphosphorylated tau, respectively (3). $\mathrm{A} \beta$ fibrils have been reported to compose SP and to be the main cause of the massive neurodegeneration observed in AD brains (5). Along with NFTs and SP, additional neuropathological characteristics of this disease include synapse loss and neuronal death (6). Synapses are a unique architecture formed by nerve cells and are considered to be the critical sites for pathogenesis in neurodegenerative diseases associated with aging $(7,8)$. The cognitive impairment in AD mainly results from synaptic loss in neurons and the deterioration of synapses usually begins at the level of dendritic spines $(9,10)$. Dendritic spines are tiny, bulbous structures protruding from the dendrites of neurons, which receive fast excitatory synaptic input in the brain. These structures compartmentalize the postsynaptic machinery and biochemical signaling molecules needed to respond to input from single presynaptic 
terminals (11). Recently, it has been reported that $\mathrm{A} \beta_{1-42}$ can decrease dendritic spine density in rat primary hippocampal neuron cultures (12). Moreover, some studies conducted in AD mouse models have shown that the trajectories of axons and dendrites were altered in the proximity of amyloid plaques, which affected synaptic integration of signals $(13,14)$.

Among $A \beta$ peptides, $A \beta_{1-42}$ is known to be the most neurotoxic (15). Its soluble oligomers may disrupt intracellular calcium homeostasis, leading to the activation of Cdk5. This proline-directed serine/threonine kinase regulates neuronal migration during development and maintains the survival and synaptic functions of mature neurons (16). It has been shown that the aberrant activity of Cdk5 induces the hyperphosphorylation of the neurofilament and microtubule-associated protein tau and serves an important role in neurodegeneration in AD (17). In addition, Cdk5 has been reported to act as an upstream regulator of mitochondrial fission during neuronal apoptosis. Inhibition of dynamin-related protein 1 (Drp1)-dependent mitochondrial fission alleviates neuronal apoptosis induced by aberrant Cdk5 expression (18). However, the underlying mechanism remains unknown. In our recent study, the mitochondrial fission protein Drp1 was identified as a direct substrate for Cdk5 (19). A $\beta_{1-42}$ stimulates Cdk5-mediated phosphorylation of Drp1 at Ser579 in cortical neurons, thereby regulating mitochondrial fission-mediated neuronal apoptosis. However, whether Cdk5-mediated Drp1 phosphorylation is also involved in $\mathrm{A} \beta_{1-42}$-induced neurodegeneration is yet to be fully elucidated.

In the present study, we hypothesized that phosphorylation of Drp1 at Ser579 may be involved in the pathogenesis of neurodegeneration. To this end, a phosphorylation-defective (phosphor-defect) mutant lentiviral vector (Lenti-Drp1-S579A) was constructed to block Drp1 phosphorylation at Ser579. After infection, the expression level of Drp1-S579A was first confirmed in primary cultures of cortical neurons. The neurite outgrowth and synapse density of cortical neurons were observed under microscope. Consistent with our previous findings (19), blockage of Drp1 phosphorylation also prevented $\mathrm{A} \beta_{1-42}$-induced cleavage of caspase- 3 and neuronal apoptosis. Taken together, the present findings demonstrated that phosphorylation of Drp1 at Ser579 served an important role in $\mathrm{A} \beta_{1-42}$-induced neurodegeneration, suggesting that this may be an effective strategy for the protection of neurons in this context.

\section{Materials and methods}

Experimental animals. A total of 26 C57BL/6 mice (6 8 weeks, 90-110 g) were purchased from Hunan SJA Laboratory Animal Co., Ltd. Mice had free access to water and food at $22-25^{\circ} \mathrm{C}$ with a 12-h light/dark cycle. The humidity was $\sim 60 \%$. All animal handling was performed in accordance with the guidelines of Animal Research Committee of Nanchang University (20). All protocols described in this article were approved by the Ethics Committee for Animal Experimentation of Nanchang University (approval no. 2018-035). All surgical procedures involving experimental animals were performed under anesthesia with $1.0 \%$ pentobarbital sodium $(50 \mathrm{mg} / \mathrm{kg}$ body weight $)$ by intraperitoneal injection and the suffering of animals was minimized to the best of our ability.
Primary cortical neuronal cultures. As previously described (19), primary cortical neuronal cultures were derived from embryonic day 14-15 fetal C57BL/6 mouse brains. In brief, the cortex isolated from embryonic mouse brains was placed in DMEM (HyClone; Cytiva) and treated with $0.125 \%$ trypsin (Beijing Solarbio Science \& Technology Co., Ltd.) and $0.004 \%$ DNase-I (Sigma-Aldrich; Merck KGaA) at $37^{\circ} \mathrm{C}$ for 15 and $10 \mathrm{~min}$, respectively. Neurons were mechanically dissociated by pipetting and were seeded on poly-L-lysine (Sigma-Aldrich; Merck KGaA)-coated glass- or plastic-bottom 35-mm culture dishes (cell density was $\sim 25,000-30,000 / 35-\mathrm{mm}$ dish for microscopic observation, or $\sim 45,000-50,000 / 35-\mathrm{mm}$ dish for western blotting). Cells were first cultured in neurobasal plating medium [neurobasal medium (Thermo Fisher Scientific, Inc.), 2\% B27 supplement, $0.5 \mathrm{mM}$ L-glutamine, $25 \mu \mathrm{M}$ L-glutamic acid, $1 \%$ penicillin-streptomycin $(\mathrm{P} / \mathrm{S})$, $10 \mathrm{mM}$ HEPES, 10\% FBS (Biological Industries)] and incubated at $37^{\circ} \mathrm{C}$ in a humidified incubator with $95 \%$ air and $5 \% \mathrm{CO}_{2}$. On the second day, neuronal cells were cultured in neurobasal feeding medium (neurobasal medium, 2\% B27 supplement, $0.5 \mathrm{mM}$ L-glutamine, $1 \% \mathrm{P} / \mathrm{S}, 10 \mathrm{mM}$ HEPES). Half the volume of media was replaced with the same volume of fresh neurobasal feeding media every 4 days.

Construction of Lenti-Drp1-S579A and infection procedure. Lenti-Drp1-S579A was constructed by Cyagen Biosciences, Inc. Briefly, Drp1-S579A site-directed mutagenesis was performed using a QuickChange kit (Agilent Technologies, Inc.) according to the manufacturer's instructions. Drp1-S579A was further subcloned into the pLV [Exp]-Puro-EF1A vector (Cyagen Biosciences, Inc.) and fused with a 6X His tag. The expression vector and package vectors ( $2 \mu \mathrm{g}$ of each vector) were co-transfected into 293T cells (the American Type Culture Collection) using Lipofectamine ${ }^{\circledR} 2000$ (Invitrogen; Thermo Fisher Scientific, Inc.) at $37^{\circ} \mathrm{C}$ for $6 \mathrm{~h}$. After $48 \mathrm{~h}$ of culture, the supernatants containing the lentivirus were harvested. Purification was then performed using ultracentrifugation at $80,000 \times \mathrm{g}$ for $2 \mathrm{~h}$ at $4^{\circ} \mathrm{C}$ and the lentiviral titer was determined.

To evaluate the infection efficiency, neurons at 3 days in vitro (DIV) were infected with the empty lentiviral vector pLV [Exp]-Puro-EF1A-mCherry at a multiplicity of infection (MOI) of 1-5. Then, 3 days after infection, the fluorescence of mCherry was used to monitor and visualize the lentiviral infection under fluorescence microscope (IX71; Olympus Corporation). The formula for calculating the infection efficiency was as follows: (The number of infected cells/total cells in the field) $x 100 \%$. To examine the expression level of the exogenous mutant Drp1-S579A, neurons at 3 DIV were infected with Lenti-Drp1-S579A or empty lentiviral vector at MOI of 1,2 and 5 at $37^{\circ} \mathrm{C}$ for $8 \mathrm{~h}$. At day 5 after infection, western blot analysis probed with anti-6His was carried out to examine the expression of phospho-defect Drp1-S579A in neurons.

Western blot analysis. Cultured neurons for western blot analysis were washed twice with PBS and lysed in RIPA lysis buffer (Beyotime Institute of Biotechnology) for $30 \mathrm{~min}$ on ice. The whole cell lysate was harvested via sonication $(20 \mathrm{KHz}$; $20 \mathrm{sec} ; 4^{\circ} \mathrm{C}$ ) in $4 \mathrm{X}$ sample buffer and the protein concentration 
was measured using a BCA protein assay reagent kit (Beyotime Institute of Biotechnology). Proteins (20 $\mu \mathrm{g}$ per lane) were separated by $10 \%$ SDS-PAGE gel and further transferred onto PVDF membranes (MilliporeSigma). After blocking with 5\% skim milk in TBS-0.1\% Tween 20 (TBST) buffer for $30 \mathrm{~min}$ at room temperature, the membranes were probed overnight at $4^{\circ} \mathrm{C}$ with the following primary antibodies: Rabbit anti-Drp1 (cat. no. 8570, 1:1,000; Cell Signaling Technology, Inc.), phospho-Drp1-Ser616 (cat. no. 3455, 1:1,000; Cell Signaling Technology, Inc.), anti-6His (cat. no. CW0083S; CWBIO), cleaved caspase-3 (cat. no. 9644, 1:1,000; Cell Signaling Technology, Inc.), rabbit anti-microtubule associated protein 2 (MAP2; cat. no. 8707, 1:1,000, Cell Signaling Technology, Inc.) and $\beta$-actin (cat. no. 4967, 1:5,000; Cell Signaling Technology, Inc.). After three washes in TBST, the membranes were incubated with HRP-conjugated secondary antibodies $(1: 5,000 ; \mathrm{CWBIO})$ for $1 \mathrm{~h}$ at room temperature. Protein bands were detected using an ECL solution (CWBIO). Densitometric analysis was performed using ImageJ software (version 1.48; National Institutes of Health).

Transfection procedures. To observe the spines on neuronal axons, the pEGFP plasmid (Clontech; $4 \mu \mathrm{g}$ per $35 \mathrm{~mm}$-well) was transfected into primary cortical neurons at 6 DIV using Lipofectamine 2000 (Invitrogen; Thermo Fisher Scientific, Inc.). According to the manufacturer's instructions, a mixture of $4 \mu \mathrm{g}$ plasmid DNA, $5 \mu \mathrm{l}$ Lipofectamine 2000 and $200 \mu \mathrm{l}$ Opti-MEM (Invitrogen; Thermo Fisher Scientific, Inc.) was incubated at room temperature for $15 \mathrm{~min}$, then added to neuronal cultures for transfection at $37^{\circ} \mathrm{C}$. After $6 \mathrm{~h}$ of incubation, the medium containing Lipofectamine was replaced with normal culture medium. Axonal spines were assessed under a fluorescence microscope (IX71; Olympus Corporation) after 18-24 h after transfection. To quantify the spines, 20 neurons in each group were randomly selected and the number of spines along $10 \mu \mathrm{m}$ of the axon was calculated using ImageJ software (version 1.48; National Institutes of Health). All experiments were repeated at least three times and the number of spines $/ 10 \mu \mathrm{m}$ of an axon was used to evaluate spine density in each group.

Immunofluorescence staining. The cortical neurons were fixed in $4 \%$ ice-cold paraformaldehyde (PFA; Beijing Solarbio Science \& Technology Co.,Ltd.) at $4^{\circ} \mathrm{C}$ for $10 \mathrm{~min}$. After fixation, cells were washed three times with $1 \mathrm{X}$ PBS and permeabilized with $0.5 \%$ Triton-X 100 and $0.5 \%$ BSA (cat. no. A2058; Sigma-Aldrich; Merck KGaA) in PBS at room temperature for $30 \mathrm{~min}$. Then, the cells were incubated with monoclonal rabbit anti-MAP2 (cat. no. 8707, 1:200; Cell Signaling Technology, Inc.) or monoclonal rabbit anti-synapsin-1 (cat. no. 6710, 1:200; Cell Signaling Technology, Inc.) antibodies at room temperature for $2 \mathrm{~h}$ and washed three times with $0.5 \%$ Triton-X 100 in $1 \mathrm{X}$ PBS. For immunofluorescence staining, cells were further incubated with Alexa Fluor 594-conjugated goat-rabbit IgG (1:200; Abcam) or Alexa Fluor 488-conjugated AffiniPure goat anti-rabbit IgG (1:200; ProteinTech Group, Inc.) antibodies in the dark at room temperature for $2 \mathrm{~h}$. The primary and secondary antibodies were diluted in $1 \mathrm{X}$ PBS with $0.5 \%$ Triton-X 100 and $0.5 \%$ BSA. Immunofluorescence signals were observed using an inverted fluorescence microscope
(IX71; Olympus Corporation; magnification, $x 40)$. To measure neurite length, 30 neurons were randomly selected and captured using fluorescence or DIC images. Neurites longer than the diameter of the soma were defined as neurites. The fluorescent signals from MAP2 and synapsin-1 immunofluorescent staining were measured to evaluate neurite length and synapse density using ImageJ software. The neurites originating from the soma were calculated as the number of primary dendrites per neuron.

Preparation of $A \beta_{1-42}$. A $\beta_{1-42}$ was purchased from Sigma-Aldrich (Merck KGaA). According to the manufacturer's instructions, the $\mathrm{A} \beta_{1-42}$ peptide was resuspended in DMSO (Beijing Solarbio Science \& Technology Co., Ltd.) to a concentration of $5 \mathrm{mM}$ and then diluted to $100 \mu \mathrm{M}$ in sterile $1 \mathrm{X}$ PBS (pH 7.4). The suspension was allowed to oligomerize for 5 days at $37^{\circ} \mathrm{C}$ and diluted to the $10 \mu \mathrm{M}$ of $\mathrm{A} \beta_{1-42}$ immediately before addition to the neuron culture medium.

Neuronal apoptosis assays. As described previously (19), mouse cortical neuronal cultures were treated with or without $10 \mu \mathrm{MA} \beta_{1-42}$ at $37^{\circ} \mathrm{C}$ for $24 \mathrm{~h}$. To examine the effect of Cdk5-mediated Drp1 phosphorylation on $\mathrm{A} \beta_{1-42}$-induced neuronal apoptosis, neurons were infected with Lenti-Drp1-S579A 3 days prior to $A \beta_{1-42}$ treatment. Then, $24 \mathrm{~h}$ after $\mathrm{A} \beta_{1-42}$ incubation, the cells were fixed with $4 \%$ ice-cold PFA at $4{ }^{\circ} \mathrm{C}$ for $10 \mathrm{~min}$. Immunofluorescence staining of MAP2 was performed to label cortical neurons. Hoechst 33258 (cat. no. 94403, Sigma-Aldrich; Merck KGaA) was used for nuclear DNA staining to evaluate chromosomal condensation and its morphological changes in neurons. After MAP2 immunofluorescence staining, the cells were further stained with Hoechst 33258 at room temperature for $5 \mathrm{~min}$, according to the manufacturer's protocol. Then, the fluorescence of MAP2 and Hoechst 33258 was detected under a fluorescence microscope (IX71; Olympus Corporation; magnification, $\mathrm{x} 40$ ). Normal neuronal nuclei were stained blue, whereas apoptotic nuclei with decreased volume and condensed chromatin were stained shiny white. Neurons with condensed and fragmented Hoechst 33258 staining were counted as dead cells.

Statistical analysis. Statistical analysis was performed using SPSS software (version 17.0; SPSS, Inc.). Data are presented as the mean \pm SEM and multiple comparisons between groups were performed using one-way ANOVA followed by post hoc Tukey's test. The comparisons between two groups were analyzed via unpaired Student's t-test. All experiments were repeated at least three times. $\mathrm{P}<0.05$ was considered to indicate a statistically significant difference.

\section{Results}

Expression level of phospho-defective Drpl-S579A in primary cortical neurons. To examine the role of Drp1 phosphorylation at Ser579 in $A \beta_{1-42}$-induced neurodegeneration and apoptosis, a lentiviral vector carrying the phospho-defective mutant Drp1-S579A was constructed. As shown in Fig. 1A, cells were infected with a lentiviral vector carrying mCherry at different multiplicity of infections (MOIs; varying from 0-5) to evaluate infection efficiency. The percentage of mCherry 
fluorescence-positive cells reached $50-70 \%$ at an MOI of 2 . Thus, in subsequent experiments, neuronal cultures were infected with Lenti-Drp1-S579A at an MOI of 2. As the phospho-defective Drp1-S579A is fused with a 6His tag in the lentiviral vector, the expression of Drp1-S579A in neurons after infection could be immunoblotted using 6His antibody. As presented in Fig. 1B, the expression of phospho-defective Drp1-S579A was detected in neurons infected with Lenti-Drp1-S579A. The exogenous mutant Drp1-S579A effectively downregulated the phosphorylation of Drp1 at Ser579 in neurons.

Lenti-Drp1-S579A protects neurons against $A \beta_{1-42}$-induced reduction in neurite length and dendritic loss. Our previous study reported that $\mathrm{A} \beta_{1-42}$ exerted an inhibitory effect on neurite outgrowth (19). The present results confirmed that primary cortical neurons at 9 DIV had long axons and well-developed dendrites. By contrast, neurons treated with $10 \mu \mathrm{M} \mathrm{A} \beta_{1-42}$ had shortened atrophic axons and dendrites (Fig. 2A). When neurons were infected with Lenti-Drp1-S579A, the deleterious effect of $A \beta_{1-42}$ on neurites was markedly weakened (Fig. 2B and C). Moreover, immunofluorescence staining of MAP2 was used to label the neuronal processes (Fig. 3A). It was found that Lenti-Drp1-S579A prevented $\mathrm{A} \beta_{1-42}$-induced axonal and dendritic atrophy in cortical neurons. $A \beta_{1-42}$ significantly decreased neurite lengths and primary dendrite numbers in neurons, which was effectively alleviated by blockage of Drp1 phosphorylation via Lenti-Drp1-S579A (Fig. 3B and C). In addition, Lenti-Drp1-S579A prevented the downregulation of MAP2 in neurons following $A \beta_{1-42}$ stimulation (Fig. 3D).

Lenti-Drp1-S579A attenuates the inhibitory effect of $A \beta_{1-42}$ on dendritic spine growth. It has been reported that $A \beta_{1-42}$ has a deleterious effect on neurite outgrowth, including dendritic spines (21). For visualization of spines, primary cortical neurons were transfected with the pEGFP plasmid at 5 DIV. GFP allowed for the identification of neurons and quantification of spines under an inverted fluorescence microscope. As shown in Fig. 4A, mature dendritic spines were observed in control neurons. Compared with control cells, neurons subjected to $10 \mu \mathrm{M} \mathrm{A} \beta_{1-42}$ exhibited a significant decrease in mature dendritic spines $(4.44 \pm 0.21$ vs. $1.45 \pm 0.26$ spines $/ 10 \mu \mathrm{m}$ for control vs. $\mathrm{A} \beta_{1-42}$, respectively; Fig. 4B). To investigate the role of Drp1 phosphorylation in $A \beta_{1-42}$-induced dendritic spine shrinkage, cortical neurons were infected with Lenti-Drp1-S579A at 3 DIV. Lenti-Drp1-S579A significantly restored dendritic spines in neurons after $A \beta_{1-42}$ treatment $\left(1.45 \pm 0.26\right.$ vs. $3.44 \pm 0.43$ spines $/ 10 \mu \mathrm{m}$ for $\mathrm{A} \beta_{1-42}$ vs. Lenti-Drp1-S579A, respectively; Fig. 4B).

Lenti-Drp1-S579A suppresses $A \beta_{1-42}$-induced synaptic loss. It is well recognized that synapses serve an important role in interneuronal communication and memory formation (3). Progressive synapse loss is one of the major hallmarks of $\mathrm{AD}$ and is the main cause of memory impairment in patients with AD (14). Several studies have shown that incubation of cortical neurons with $A \beta_{1-42}$ decreases synapse number $(22,23)$. Immunofluorescence staining of synapsin was conducted to detect synapses in neurons. As shown in Fig. 5A, synapsin-1-positive puncta were prevalent
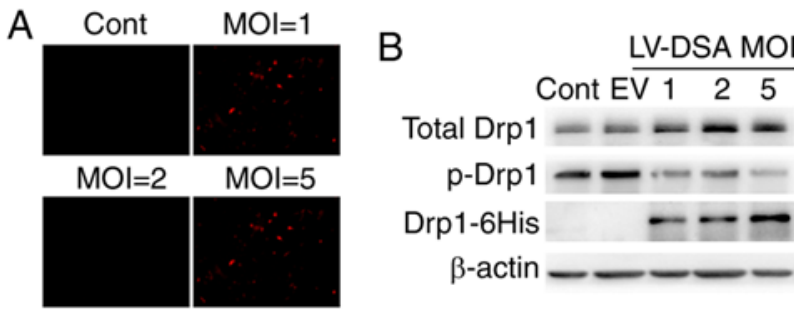

Figure 1. Lentiviral infection efficiency and expression level of Drp1-S579A in primary cortical neurons. (A) The lentiviral infection efficiency in neurons at different MOI. Primary cortical neurons were infected with lentiviral vector carrying mCherry at a MOI of 0-5. Infection efficiency was monitored via mCherry fluorescence in neurons (magnification, x10). Scale bar, $50 \mu \mathrm{m}$. (B) Expression of phosphorylation-defect Drp1 in neurons infected with Lenti-Drp1-S579A. Neurons were infected with Lenti-Drp1-S579A at a MOI of 0-5 or empty lentiviral vector at MOI of 5. Then, 3 days later, cells were harvested for western blot analysis to detect the expression level of 6His-tagged Drp1-S579A, phosphorylation of Drp1 at Ser579 and total-Drp1. $\beta$-actin was used as an endogenous control. EV, empty lentiviral vector; LV-DSA, lenti-Drp1-S579A; Drp1, dynamin-related protein 1; MOI, multiplicity of infection; Cont, control; p-, phosphorylated.

A
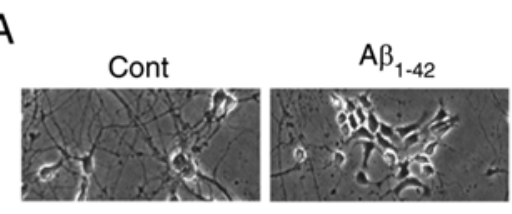

Drp1-
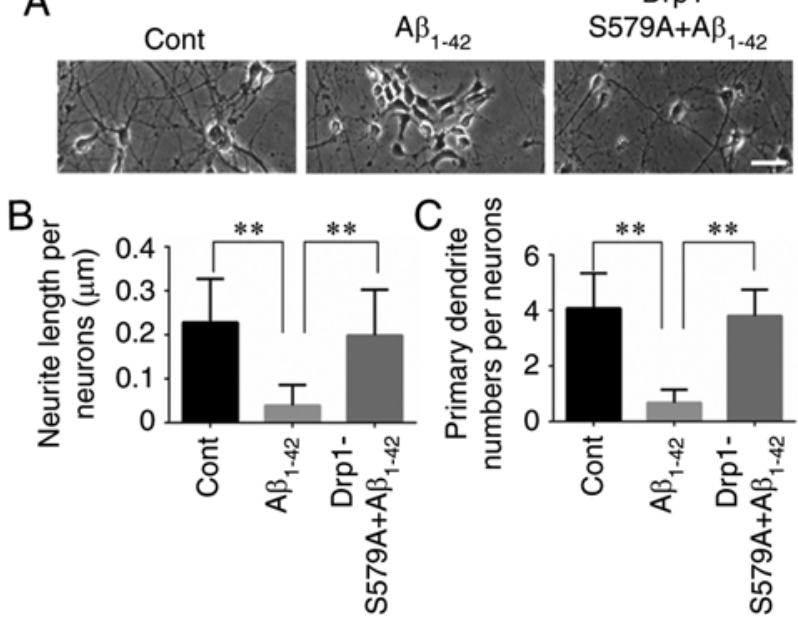

$\mathrm{C}_{\oplus} \stackrel{0}{\check{0}}$

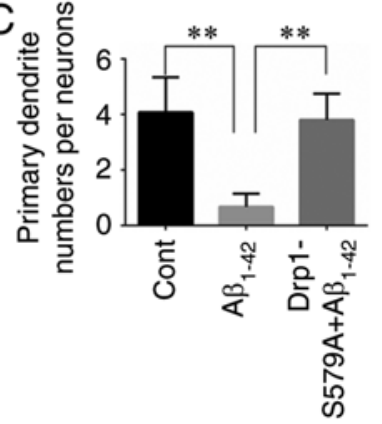

Figure 2. Effect of Lenti-Drp1-S579A on A $\beta_{1-42}$-induced neurite alterations under phase-contrast microscope. (A) Representative images of primary cortical neurons under phase-contrast microscope. Neurons were incubated with or without $10 \mu \mathrm{M} \mathrm{A} \beta_{1-42}$ for $24 \mathrm{~h}$. To examine the effect of phosphorylation-defect Drp1 on $\mathrm{A} \beta_{1-42}$-induced alterations in neurons, Lenti-Drp1-S579A was infected to cells at 3 days prior to $A \beta_{1-42}$ stimulation (magnification, 20). Scale bar, $50 \mu \mathrm{m}$. Effect of Lenti-Drp1-S579A on A $\beta_{1-42}$-induced neuronal alterations. The graphs indicate (B) neurite length and (C) dendrite number in primary cortical neurons in each group. ${ }^{* *} \mathrm{P}<0.01$. Drp1, dynamin-related protein 1 ; Cont, control; $A \beta$, amyloid $\beta$.

in neuronal processes in the control group. By contrast, synapsin-1 staining displayed a localization in the soma and decreased along neuronal processes after $\mathrm{A} \beta_{1-42}$ incubation, indicating reduced synapse density ( 1.00 vs. $30.11 \pm 5.80 \%$ for control vs. $A \beta_{1-42}$, respectively; Fig. 5B). Moreover, infection with Lenti-Drp1-S579A restored synapsin-1 staining along neuronal processes $\left(30.11 \pm 5.80\right.$ vs. $100.90 \pm 13.66 \%$ for $\mathrm{A} \beta_{1-42}$ vs. Lenti-Drp1-S579A, respectively; Fig. 5B).

Lenti-Drp1-S579A protects neurons against A $\beta_{1-42}$-induced apoptosis. Exposure of cultured neurons to submicromolar concentrations of $A \beta_{1-42}$ may induce direct neurotoxicity (24). 


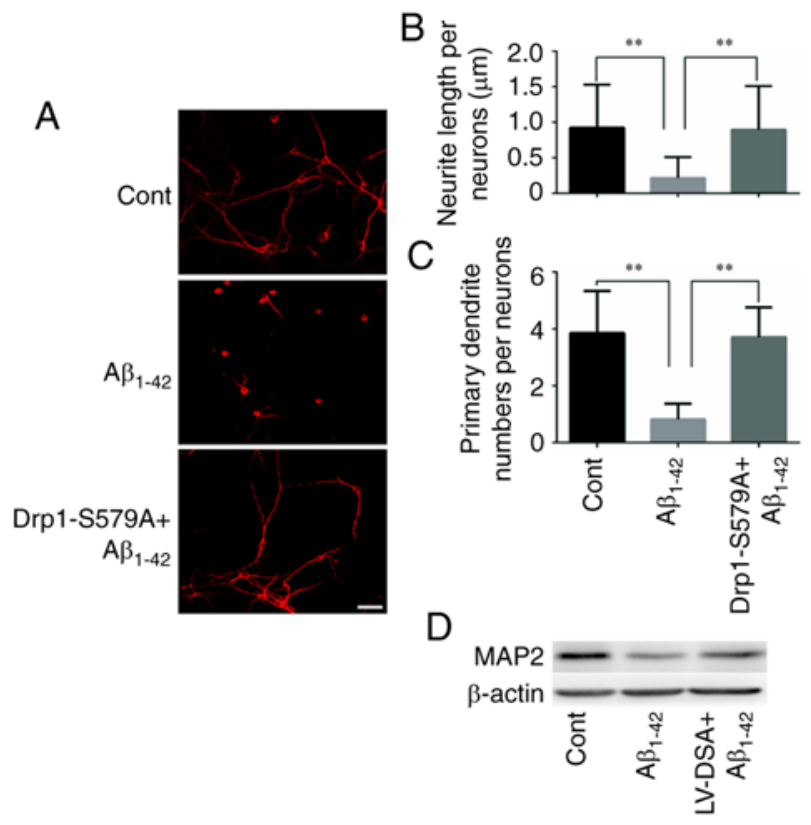

Figure 3. Effect of Lenti-Drp1-S579A on $\mathrm{A} \beta_{1-42}$-induced neurite alterations under fluorescence microscope. (A) Representative images of primary cortical neurons under fluorescence microscope. Fluorescence immunostaining of MAP2 was conducted in neurons after the indicated treatments. Neurite morphology was detected under a fluorescence microscope (magnification, $\mathrm{x} 20$ ). Scale bar, $50 \mu \mathrm{m}$. Effect of Lenti-Drp1-S579A on A $\beta_{1-42}$-induced neurite atrophy. The graphs indicate (B) neurite length and (C) dendrite number in primary cortical neurons in each group. ${ }^{* *} \mathrm{P}<0.01$. (D) Lenti-Drp1-S579A inhibited $A \beta_{1-42}$-induced decrease of MAP2 expression in neurons. $\beta$-actin was used as an endogenous control. LV-DSA, lenti-Drp1-S579A; MAP2, microtubule associated protein 2; Cont, control; $\mathrm{A} \beta$, amyloid $\beta$; Drp1, dynamin-related protein 1 .
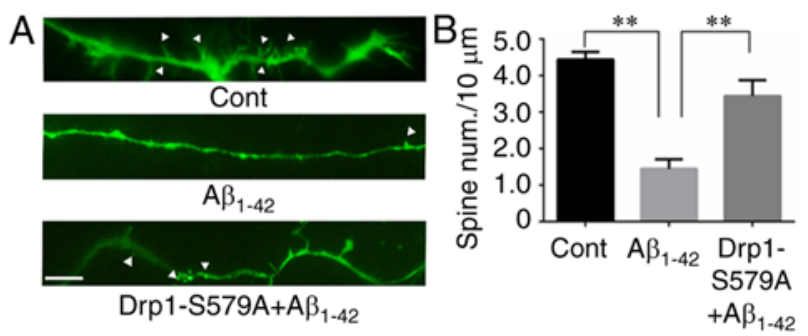

Figure 4. Lenti-Drp1-S579A prevents dendritic spine reduction induced by $\mathrm{A} \beta_{1-42}$. (A) Representative fluorescence images of dendritic spines. GFP was transfected to neurons to outline neurite morphology. White arrows indicate the dendritic spines on neuronal processes (magnification, x60). Scale bar, $5 \mu \mathrm{m}$. (B) The quantitative analysis of dendritic spines. The graph indicates the spine number $/ 10 \mu \mathrm{m}$ neuronal processes in each group. The data are presented as the mean \pm SEM of at least three independent experiments ${ }^{* *} \mathrm{P}<0.01$. Cont, control; $\mathrm{A} \beta$, amyloid $\beta$; Drp1, dynamin-related protein 1 .

Here, the effect of Lenti-Drp1-S579A on A $\beta_{1-42}$-induced apoptosis was examined. To detect neuronal apoptosis, the cells were stained with Hoechst 33258 and MAP2 antibody. As presented in Fig. 6A, MAP2-positive cells with condensed Hoechst 33258 staining were counted as apoptotic neurons. Compared with the control group, $A \beta_{1-42}$ stimulation significantly increased the number of apoptotic neurons $\left(5.00 \pm 1.08\right.$ vs. $85.75 \pm 6.14 \%$ for control vs. $\mathrm{A} \beta_{1-42}$, respectively). Furthermore, infection with Lenti-Drp1-S579A efficiently alleviated $A \beta_{1-42}$-induced apoptosis (85.75 \pm 6.14 vs.
$39.25 \pm 2.25 \%$ for $A \beta_{1-42}$ vs. Lenti-Drp1-S579A, respectively; Fig. 6B).

Next, the cleavage of caspase- 3 was examined using western blot analysis in neurons after $A \beta_{1-42}$ exposure. The results demonstrated that $A \beta_{1-42}$ significantly stimulated the cleavage of caspase- 3 in neurons ( 1.00 vs. $1.73 \pm 0.08$ for control vs. $A \beta_{1-42}$, respectively), which was efficiently prevented by Lenti-Drp1-S579A (1.73 \pm 0.08 vs. $1.14 \pm 0.15$ for $\mathrm{A} \beta_{1-42}$ vs. Lenti-Drp1-S579A, respectively) (Fig. 7).

\section{Discussion}

$\mathrm{AD}$ is the most common neurodegenerative disease, with $\mathrm{A} \beta$ plaques as one of the major pathological hallmarks (25). In several AD mouse models, $A \beta$ peptide deposition in the brain has been reported to be associated with various neuronal abnormalities, including the dystrophic neurites (26), dendritic spine loss (27), development of synaptic dysfunction (28) and abnormal neuronal firing (29). The variety of neuronal deficits associated with the deposition of $\mathrm{A} \beta$ peptides likely contributes to cognitive decline and memory loss in patients with $\mathrm{AD}(8,30)$. It is considered that the neurotoxicity of $\mathrm{A} \beta_{1-42}$ is responsible for neurodegeneration in the AD brain (31). In addition, aberrant activity of Cdk5 is also involved in the $\mathrm{A} \beta$-evoked neurotoxic cascade (32). Cdk5 serves a vital role in the development of the central nervous system, maintenance of synaptic plasticity and neuronal apoptosis in response to stress (33). Moreover, it acts as an upstream regulator of Drp1-dependent mitochondrial fission during neuronal apoptosis (18). However, the underlying mechanism remains to be elucidated.

In our previous study, the mitochondrial fission protein Drp1 was identified as the direct substrate of Cdk5 and $A \beta_{1-42}$ effectively induced Cdk5-meidated Drp1 phosphorylation at Ser579. Furthermore, it was observed that Cdk5-mediated Drp1 phosphorylation at Ser579 was involved in $\mathrm{A} \beta_{1-42}$-induced mitochondrial fission and neuronal apoptosis (19), indicating that blockage of this process may be a possible strategy to prevent $A \beta_{1-42}$-induced neurodegeneration. To prove this hypothesis, the current study constructed a lentiviral vector carrying phospho-defective Drp1-S579A. The expression level of total Drp1 and exogenous mutant Drp1-S579A in neurons were detected using western blotting with Drp1 antibody and anti-6His antibody, respectively. Since the affinity of different antibodies is distinct, it is inappropriate to compare the expression of endogenous and exogenous mutant Drp1 by the density of these blot bands. Notably, the mutant Drp1-S579A effectively decreased the level of Drp1 phosphorylation at Ser579 in neurons. This result was consistent with that of a previous study (34), in which the phosphorylation site at Ser579 was reported to be necessary for the GTPase activity of Drp1. Although the phospho-defective Drp1-S579A may have the same ability to interact with Fis1 as wild-type Drp1, mitochondrial fission cannot be mediated by the mutant Drpl without GTPase activity (34). In the present study, it remains unclear how the exogenous phospho-defective Drp1-S579A had an inhibitory effect on the phosphorylation of Drp1-Ser579 in neurons. Whether the mutant Drp1-S579A may competitively inhibit Cdk5-mediated wild-type Drp1 phosphorylation remains to be determined. 
A
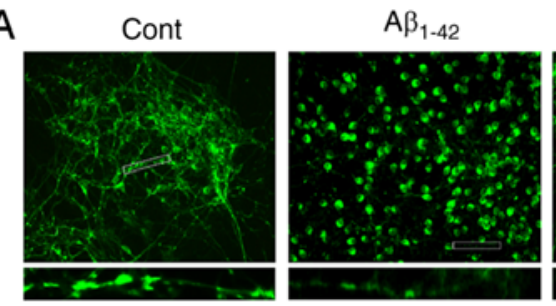

Drp1-S579A+A $\beta_{1-42}$

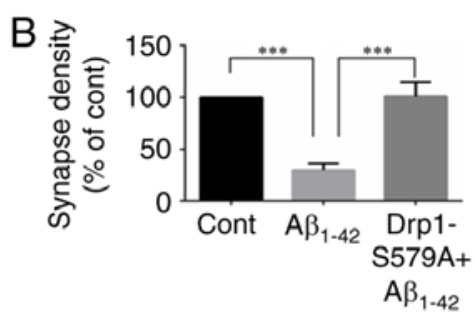

Figure 5. Lenti-Drp1-S579A inhibits A $\beta 1$-42-induced synapse loss in cortical neurons. (A) Neuronal synapses were evaluated via immunofluorescence staining of synapsin-1. The upper panels are the representative images of immunofluorescence staining of synapsin-1 in each group (magnification, $\mathrm{x} 40 ; \mathrm{x} 200$ in zoomed images). Scale bar, $50 \mu \mathrm{m}$. The below panels show synapsin-1 immunostaining with higher magnification in the inserted boxes. (B) The synapsin-1-positive fluorescence intensity along neuronal processes was determined as synapse density. The graph indicates the relative synapse density along neuronal processes in each group. ${ }^{* * * *} \mathrm{P}<0.001$. Cont, control; A $\beta$, amyloid $\beta$; Drp1, dynamin-related protein 1.
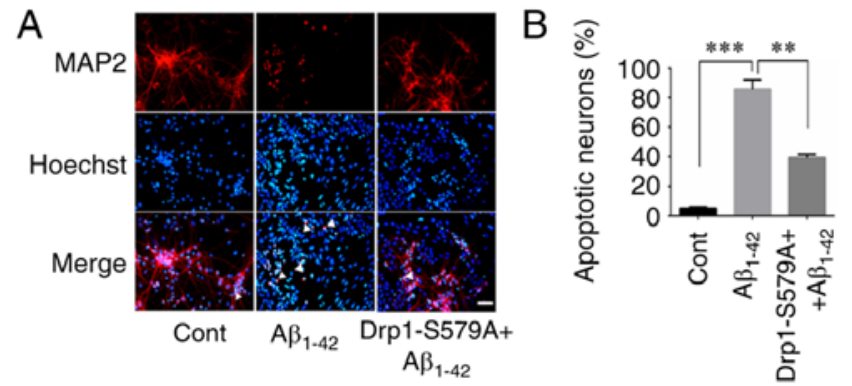

Figure 6. Lenti-Drp1-S579A attenuates $A \beta_{1-42}$-induced neuronal apoptosis (A) Neurons were double-stained with MAP2 (Red) and Hoechst 33258 (Blue) after the indicated treatments. White arrows indicate the apoptotic neurons with condensed Hoechst 33258 (magnification, x40). Scale bar $50 \mu \mathrm{m}$. (B) The graph shows the percentage of apoptotic neurons in each group. The data are presented as the mean \pm SEM of at least three independent experiments. ${ }^{* * *} \mathrm{P}<0.01,{ }^{* * *} \mathrm{P}<0.001$. Cont, control; $\mathrm{A} \beta$, amyloid $\beta$; Drp1, dynamin-related protein 1; MAP2, microtubule associated protein 2 .
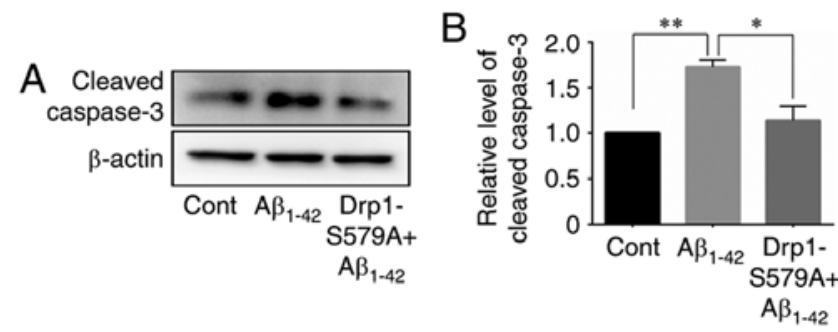

Figure 7. Lenti-Drp1-S579A alleviates $A \beta_{1-42}$-induced the cleavage of caspase-3 in neurons. (A) The expression level of cleaved caspase-3 was examined in neurons after the indicated treatments. $\beta$-actin was used as an endogenous control. (B) Relative expression level of cleaved caspase-3 was indicated as a normalization of the ratio of cleaved caspase- $3 / \beta$-actin in each sample to the $A \beta_{1-42}$ group. The data are presented as the mean \pm SEM of at least three independent experiments. ${ }^{*} \mathrm{P}<0.05,{ }^{* *} \mathrm{P}<0.01$. Cont, control; $\mathrm{A} \beta$, amyloid $\beta$; Drp1, dynamin-related protein 1 .
Next, the effect of Lenti-Drp1-S579A on $\mathrm{A} \beta_{1-42}$-induced neurite atrophy, synapse loss was examined. The results demonstrated that the $A \beta_{1-42}$-mediated decrease in neurite length, synapse number and density of dendrites and dendritic spines was mostly prevented by the blockage of Drp1 phosphorylation at Ser579, but it does not suggest that Lenti-Drp1-S579A completely blocked all of the $A \beta_{1-42}$-induced neuronal injury. Moreover, the $A \beta_{1-42}$-induced neuronal apoptosis was still detected in Drp1-S579A $+A \beta_{1-42}$ group. Neuronal apoptosis might be a later event in the $A \beta_{1-42}$-induced degeneration of dendritic growth and loss of spines and synapses and the inhibitory effect of Lenti-Drp1-S579A on $\mathrm{A} \beta_{1-42}$-induced neurodegeneration is greater than neuronal apoptosis. Taken together, the current data support the important role of Cdk5-mediated Drp1 phosphorylation at Ser579 in $A \beta_{1-42}$-induced neurodegeneration. In addition, the decrease in neurite length, synapse number and density of dendrite and dendrite spine is the common characteristic of neurodegeneration. Therefore, the present study indicates that blockage of Drp1 phosphorylation at Ser579 efficiently protects neurons against $\mathrm{A} \beta_{1-42}$-induced neurodegeneration.

On the other hand, it has been well documented that mitochondrial dysfunction serves a crucial role in various neurodegenerative diseases, including AD, Parkinson's disease and Huntington disease (35-37). Mitochondria are highly dynamic organelles, with their morphology changing frequently via fission and fusion events. Some large GTPases have been identified as regulators of mitochondrial fission and fusion. Mitochondrial outer membrane fission is mediated by Drp1 and mitochondrial fission protein (Fis1) (38). Optic atrophy and mitofusins regulate mitochondrial inner or outer membrane fusion, respectively (39). Several studies have reported abnormalities in mitochondrial function and dynamics in neurodegenerative diseases $(14,40,41)$. Impaired balance of mitochondrial fusion and fission has been observed in the hippocampal tissue of patients with AD (41). Furthermore, inhibition of Drp1-mediated mitochondrial fission protects dopaminergic neurons against neurite loss and apoptosis following mitochondrial stress (42). Consistent with previous studies, the mutant Drp1-S579A alleviated $A \beta_{1-42}$-induced cleavage of caspase- 3 and neuronal apoptosis. In addition, the post translational modifications of Drp1 are closely associated with its activity and mitochondrial fission, including phosphorylation, SUMOylation and nitrosylation (43-48). Calmodulin-dependent protein kinase Ia phosphorylates Drp1 at Ser616 and triggers mitochondrial fission by promoting the interaction between Drpl and Fis1 (43). GSK-3-mediated phosphorylation of Drp1 at Ser40 and Ser44 enhances the GTPase activity of Drp1 and induces mitochondrial fragmentation (45). In addition, S-nitrosylation of Drp1 also bridges excessive mitochondrial fission with neuronal injury during neurodegeneration (48). Therefore, it would be useful to further examine whether there is a crosstalk between Cdk5-mediated Drp1 phosphorylation and S-nitrosylation of the same protein in neurodegeneration.

The present study indicated that blockage of Drp1 phosphorylation at Ser579 protected cortical neurons against $\mathrm{A} \beta_{1-42}$-induced degeneration and apoptosis. However, there are some limitations in the present study. First, the effect of lenti-Drp1-S579A on neurodegeneration was only examined in primary cultured cortical neurons. It is still unclear whether 
blockage of Drp1 phosphorylation at Ser579 prevents neurodegeneration in vivo. Therefore, it is necessary to examine the effect of lenti-Drp1-S579A on neurodegeneration and neuronal apoptosis in AD animal model. Second, the functional consequences of Cdk5-mediated Drp1 phosphorylation remain controversial. It has been reported that Cdk5-mediated phosphorylation of Drp1 at Ser616 inhibits mitochondrial fission during neuronal maturation (47). By contrast, Jahani-Asl et al (47) and our previous study (19) revealed that $\mathrm{Cdk} 5$-mediated phosphorylation of Drp1 at Ser616 or Ser579 (the same conserved serine residue as Ser585 in different Drp1 isoforms) stimulates mitochondrial fission in neurons after exposure to N-methyl-d-aspartate or $\mathrm{A} \beta_{1-42}$, respectively. Although it is possible that the opposite effect of Cdk5-mediated Drp1 phosphorylation at the same conserved serine residue may be explained by the level of maturity of neurons, it would be useful to further investigate the underlying mechanism.

In conclusion, it was suggested that the atrophy of neuronal processes, synapse loss and neuronal apoptosis are the characteristic alterations in $\mathrm{AD}$, a common neurodegenerative disease and that mitochondrial dysfunction is involved in the pathogenesis of AD. In our previous study, the Cdk5-mediated phosphorylation of Drp1 at Ser579 was found to regulate $A \beta_{1-42}$ induced mitochondrial fission and neuronal apoptosis (19). Thus, it was necessary to further investigate the role of Drp1 phosphorylation at Ser579 in neurodegeneration. The current study constructed a lentiviral vector carrying phospho-defect Drp1-S579A. It was found that inhibition of Drp1 phosphorylation at Ser579 by Lenti-Drp1-S579A efficiently attenuated the $A \beta_{1-42}$-mediated decrease in neurite length, synapse number, density of dendrites and dendritic spines and neuronal apoptosis. This suggests the involvement of Drp1 phosphorylation at Ser579 in neurodegeneration in $\mathrm{AD}$ and corroborates the potential of blocking this process to prevent neurodegeneration.

\section{Acknowledgements}

Not applicable.

\section{Funding}

This work was supported by National Natural Science Foundation of China (grant nos. 81660159, 81660607 and 82060177), Jiangxi Province Natural Science Foundation (grant no. 20192BAB205050), Jiangxi Province Major discipline and academic leaders training Program Project (grant no. 20172BCB22028), the Research Fund for Jiangxi Geriatric Clinical Medical Research Center (grant no. 2020BCG74003) and Jiangxi Province Key Laboratory of Experimental Animals (grant no. 20192BCD40003), Key Research and Development Program of Jiangxi Province (grant no. 20192BBG70049) and the Research Fund from Jiangxi Administration of Traditional Chinese Medicine (grant no. 2019A084).

\section{Availability of data and materials}

The datasets used and/or analyzed during the current study are available from the corresponding author on reasonable request.

\section{Authors' contributions}

XJH and XHQ conceived and designed the research. DX and ZJY conducted the cellular and molecular biological experiments. PY, LPJ and YTO raised C57BL/6 mice and conducted neuronal primary culture. TY, JHS, QGL and YYW performed fluorescent microscopy. DX, PY and QGL analysed and interpreted the data. XJH and XHQ confirm the authenticity of all the raw data. XJH and LPJ wrote the manuscript. All authors read and approved the final manuscript.

\section{Ethics approval and consent to participate}

The animal experiments in the present study were approved by the Animal Research Ethics Committee of Affiliated People's Hospital of Nanchang University (approval no. 2018-035).

\section{Patient consent for publication}

Not applicable.

\section{Competing interests}

The authors declare that they have no competing interests.

\section{References}

1. Reddy PH, Manczak M, Mao P, Calkins MJ, Reddy AP and Shirendeb U: Amyloid-beta and mitochondria in aging and Alzheimer's disease: Implications for synaptic damage and cognitive decline. J Alzheimers Dis 20 (Suppl 2): S499-S512, 2010.

2. Mattson MP: Pathways towards and away from Alzheimer's disease. Nature 430: 631-639, 2004.

3. Vaz M and Silvestre S: Alzheimer's disease: Recent treatment strategies. Eur J Pharmacol 887: 173554, 2020.

4. Reddy PH, Manczak M and Yin X: Mitochondria-division inhibitor 1 protects against amyloid- $\beta$ induced mitochondrial fragmentation and synaptic damage in Alzheimer's disease. J Alzheimers Dis 58: 147-162, 2017.

5. Pozueta J, Lefort R and Shelanski ML: Synaptic changes in Alzheimer's disease and its models. Neuroscience 251: 51-65, 2013.

6. Koffie RM, Hyman BT and Spires-Jones TL: Alzheimer's disease: Synapses gone cold. Mol Neurodegener 6: 63, 2011.

7. DeKosky ST and Scheff SW: Synapse loss in frontal cortex biopsies in Alzheimer's disease: Correlation with cognitive severity. Ann Neurol 27: 457-464, 1990.

8. Terry RD, Masliah E, Salmon DP, Butters N, DeTeresa R, Hill R, Hansen LA and Katzman R: Physical basis of cognitive alterations in Alzheimer's disease: Synapse loss is the major correlate of cognitive impairment. Ann Neurol 30: 572-580, 1991.

9. Harris KM and Kater SB: Dendritic spines: Cellular specializations impartingboth stability and flexibility to synaptic function. Annu Rev Neurosci 17: 341-371, 1994.

10. Carlisle HJ and Kennedy MB: Spine architecture and synaptic plasticity. Trends Neurosci 28: 182-187, 2005.

11. Serrano-Pozo A, Frosch MP, Masliah E and Hyman BT: Neuropathological alterations in Alzheimer disease. Cold Spring Harb Perspect Med 1: a006189, 2011.

12. Ryu J, Hong BH, Kim YJ, Yang EJ, Choi M, Kim H, Ahn S, Baik TK, Woo RS and Kim HS: Neuregulin-1 attenuates cognitive function impairments in a transgenic mouse model of Alzheimer's disease. Cell Death Dis 7: e2117, 2016.

13. Colgan LA and Yasuda R: Plasticity of dendritic spines: Subcompartmentalization of signaling. Annu Rev Physiol 76: 365-385, 2014

14. Jankowsky JL and Zheng H: Practical considerations for choosing a mouse model of Alzheimer's disease. Mol Neurodegener 12: 89, 2017. 
15. Barage SH and Sonawane KD: Amyloid cascade hypothesis: Pathogenesis and therapeutic strategies in Alzheimer's disease. Neuropeptides 52: 1-18, 2015.

16. Shah K and Lahiri DK: Cdk5 activity in the brain-multiple paths of regulation. J Cell Sci 127: 2391-2400, 2014.

17. Nguyen MD, Larivière RC and Julien JP: Deregulation of Cdk5 in a mouse model of ALS: Toxicity alleviated by perikaryal neurofilament inclusions. Neuron 30: 135-147, 2001.

18. Meuer K, Suppanz IE, Lingor P, Planchamp V, Göricke B, Fichtner L, Braus GH, Dietz GP, Jakobs S, Bähr $M$ and Weishaupt JH: Cyclin-dependent kinase 5 is an upstream regulator of mitochondrial fission during neuronal apoptosis. Cell Death Differ 14: 651-661, 2007.

19. Guo MY, Shang L, Hu YY, Jiang LP, Wan YY, Zhou QQ, Zhang K, Liao HF, Yi JL and Han XJ: The role of Cdk5-mediated Drpl phosphorylation in $\mathrm{A} \beta_{1-42}$ induced mitochondrial fission and neuronal apoptosis. J Cell Biochem 119: 4815-4825, 2018.

20. Yan M, Guo A, Chen P, Jing H, Ren D, Zhong Y, Wu Y, Fei E, Lai X, Zou S and Wang S: LRP4 LDL $\alpha$ repeats of astrocyte enhance dendrite arborization of the neuron. Mol Brain 13: 166, 2020.

21. Kandimalla R, Manczak M, Yin X, Wang R and Reddy PH: Hippocampal phosphorylated tau induced cognitive decline, dendritic spine loss and mitochondrial abnormalities in a mouse model of Alzheimer's disease. Hum Mol Genet 27 30-40, 2018

22. Ingelsson M, Fukumoto $\mathrm{H}$, Newell KL, Growdon JH, Hedley-Whyte ET, Frosch MP, Albert MS, Hyman BT and Irizarry MC: Early Abeta accumulation and progressive synaptic loss, gliosis and tangle formation in AD brain. Neurology 62: 925-931, 2004.

23. Lista $\mathrm{S}$ and Hampel H: Synaptic degeneration and neurogranin in the pathophysiology of Alzheimer's disease. Expert Rev Neurother 17: 47-57, 2017.

24. Deshpande A, Mina E, Glabe C and Busciglio J: Different conformations of amyloid beta induce neurotoxicity by distinct mechanisms in human cortical neurons. J Neurosci 26: 6011-6018, 2006.

25. Cummings BJ, Su JH, Geddes JW, Van Nostrand WE, Wagner SL, Cunningham DD and Cotman CW: Aggregation of the amyloid precursor protein within degenerating neurons and dystrophic neurites in Alzheimer's disease. Neuroscience 48: 763-777, 1992

26. Grutzendler J, Helmin K, Tsai J and Gan WB: Various dendritic abnormalities are associated with fibrillar amyloid deposits in Alzheimer's disease. Ann N Y Acad Sci 1097: 30-39, 2007.

27. Tsai J, Grutzendler J, Duff K and Gan WB: Fibrillar amyloid deposition leads to local synaptic abnormalities and breakage of neuronal branches. Nat Neurosci 7: 1181-1183, 2004.

28. Walsh DM, Klyubin I, Fadeeva JV, Cullen WK, Anwyl R, Wolfe MS, Rowan MJ and Selkoe DJ: Naturally secreted oligomers of amyloid beta protein potently inhibit hippocampal long-term potentiation in vivo. Nature 416: 535-539, 2002.

29. Busche MA, Eichhoff G, Adelsberger H, Abramowski D, Wiederhold KH, Haass C, Staufenbiel M, Konnerth A and Garaschuk O: Clusters of hyperactive neurons near amyloid plaques in a mouse model of Alzheimer's disease. Science 321: 1686-1689, 2008.

30. Masliah E, Ellisman M, Carragher B, Mallory M, Young S, Hansen L, DeTeresa R and Terry RD: Three-dimensional analysis of the relationship between synaptic pathology and neuropil threads in Alzheimer disease. J Neuropathol Exp Neurol 51: 404-414, 1992.

31. Postuma RB, He W, Nunan J, Beyreuther K, Masters CL, Barrow CJ and Small DH: Substrate-bound beta-amyloid peptides inhibit cell adhesion and neurite outgrowth in primary neuronal cultures. J Neurochem 74: 1122-1130, 2000.
32. Wilkaniec A, Gassowska-Dobrowolska M, Strawski M, Adamczyk A and Czapski GA: Inhibition of cyclin-dependent kinase 5 affects early neuroinflammatory signalling in murine model of amyloid beta toxicity. J Neuroinflammation 15: 1, 2018.

33. Huang E, Qu D, Zhang Y, Venderova K, Haque ME, Rousseaux MW, Slack RS, Woulfe JM and Park DS: The role of Cdk5-mediated apurinic/apyrimidinic endonuclease 1 phosphorylation in neuronal death. Nat Cell Biol 12: 563-571, 2010.

34. Taguchi N, Ishihara N, Jofuku A, Oka T and Mihara K: Mitotic phosphorylation of dynamin-related GTPase Drp1 participates in mitochondrial fission. J Biol Chem 282: 11521-11529, 2007.

35. Zhang WY, Gu ZL, Liang ZQ and Qin ZH: Mitochondrial dysfunction and Huntington disease. Neurosci Bull 22: 129-136, 2006.

36. Baloyannis SJ: Mitochondrial alterations in Alzheimer's disease. J Alzheimers Dis 9: 119-126, 2006.

37. Cho DH, Nakamura T, Fang J, Cieplak P, Godzik A, Gu Z and Lipton SA: S-nitrosylation of Drp1 mediates beta-amyloid-related mitochondrial fission and neuronal injury. Science 324: 102-105, 2009.

38. Kim H, Scimia MC, Wilkinson D, Trelles RD, Wood MR, Bowtell D, Dillin A, Mercola M and Ronai ZA: Fine-tuning of Drp1/Fis1 availability by AKAP121/Siah2 regulates mitochondrial adaptation to hypoxia. Mol Cell 44: 532-544, 2011.

39. Chandhok G, Lazarou M and Neumann B: Structure, function and regulation of mitofusin-2 in health and disease. Biol Rev Camb Philos Soc 93: 933-949, 2018.

40. Yan QW, Zhao N, Xia J, Li BX and Yin LY: Effects of treadmill exercise on mitochondrial fusion and fission in the hippocampus of APP/PS1 mice. Neurosci Lett 701: 84-91, 2019.

41. Wang X, Su B, Lee HG, Li X, Perry G, Smith MA and Zhu X: Impaired balance of mitochondrial fission and fusion in Alzheimer's disease. J Neurosci 29: 9090-9103, 2009.

42. Qi X, Qvit N, Su YC and Mochly-Rosen D: A novel Drp1 inhibitor diminishes aberrant mitochondrial fission and neurotoxicity. J Cell Sci 126: 789-802, 2013.

43. Han XJ, Lu YF, Li SA, Kaitsuka T, Sato Y, Tomizawa K, Nairn AC, Takei K, Matsui H and Matsushita M: CaM kinase I alpha-induced phosphorylation of Drp1 regulates mitochondrial morphology. J Cell Biol 182: 573-585, 2008

44. Cereghetti GM, Stangherlin A, Martins de Brito O, Chang CR, Blackstone C, Bernardi P and Scorrano L: Dephosphorylation by calcineurin regulates translocation of Drp1 to mitochondria. Proc Natl Acad Sci USA 105: 15803-15808, 2008.

45. Yan J, Liu XH, Han MZ, Wang YM, Sun XL, Yu N, Li T, Su B and Chen ZY: Blockage of GSK3 $\beta$-mediated Drp1 phosphorylation provides neuroprotection in neuronal and mouse models of Alzheimer's disease. Neurobiol Aging 36: 211-227, 2015.

46. Cho B, Cho HM, Kim HJ, Jeong J, Park SK, Hwang EM, Park JY, Kim WR, Kim H and Sun W: CDK5-dependent inhibitory phosphorylation of Drp1 during neuronal maturation. Exp Mol Med 46: e105, 2014.

47. Jahani-Asl A, Huang E, Irrcher I, Rashidian J, Ishihara N, Lagace DC, Slack RS and Park DS: CDK 5 phosphorylates DRP1 and drives mitochondrial defects in NMDA-induced neuronal death. Hum Mol Genet 24: 4573-4583, 2015.

48. Nakamura T, Cieplak P, Cho DH, Godzik A and Lipton SA: S-nitrosylation of Drpl links excessive mitochondrial fission to neuronal injury in neurodegeneration. Mitochondrion 10: 573-578, 2010.

This work is licensed under a Creative Commons Attribution-NonCommercial-NoDerivatives 4.0 International (CC BY-NC-ND 4.0) License. 